\title{
On the Numerical Accuracy of Electromagnetic Transient Simulation with Power Electronics
}

\author{
Jeroen Tant, Member, IEEE, and Johan Driesen, Senior Member, IEEE
}

\begin{abstract}
This paper investigates the numerical accuracy of currently employed methods for electromagnetic transient simulation with power electronics. Existing tools often employ the second-order accurate trapezoidal method for numerical integration. However, solution techniques employed to facilitate ideal power electronic switches do not always preserve this second-order accuracy at switch events. As a result, the step size required to achieve the desired level of accuracy is often smaller than expected, affecting simulation speed. Therefore, this paper aims to assess and improve the trade-off between step size and accuracy. First, currently employed solution techniques are reviewed by deriving asymptotic estimates of the rates at which approximation errors decrease as the step size reduces. Afterwards, a benchmark method is proposed which preserves second-order accuracy at switch events. The tools EMTP-RV and PSCAD are compared with this benchmark method for elementary circuit examples. The results demonstrate the potential to improve accuracy, by several orders of magnitude in some cases, without increasing the number of linear systems to be solved.
\end{abstract}

Index Terms-Electromagnetic transients, power electronics, numerical simulation, accuracy.

\section{INTRODUCTION}

$\mathbf{S}$ IMULATION tools are widely applied to study electromagnetic transients in transmission and distribution grids [1]-[6]. In such studies, it is often of interest to analyze the switching behavior of power electronic equipment such as high-voltage dc (HVDC) converters and flexible ac transmission system (FACTS) devices [7]-[10]. To accommodate this, most tools support idealized models of power electronic switching devices, realized with small on-resistances and large off-resistances [11]-[13].

The support of idealized switches requires specialized solution techniques [7], [12], [14]-[16]. Table I lists literature on techniques currently used in selected tools for (nonrealtime) electromagnetic transient simulation. All listed tools except XTAP use the trapezoidal (TR) method for numerical integration. XTAP uses 2s-DIRK, an $L$-stable method of order two. The anticipated second-order accuracy of these methods is, however, compromised at switch events because approximations of lower order are involved, for instance due to the use of linear interpolation for event localization [17], due to the use of backward Euler (BE) steps for the suppression of numerical oscillations [18], or in the reinitialization process after switching [19]. Consequently, simulations with switch events are generally less accurate than simulations without

This work was partially supported by the Belgian Science Policy Office Grant IAP P7/02. The work of J. Tant has been supported by a Ph.D. fellowship from the Research Foundation - Flanders (FWO).

J. Tant and J. Driesen are both with ELECTA, Department of Electrical Engineering, KU Leuven, Leuven, Belgium, and Energyville, Genk, Belgium (e-mail: jeroen.tant@esat.kuleuven.be; johan.driesen@esat.kuleuven.be).
TABLE I

TOOLS FOR ELECTROMAGNETIC TRANSIENT SIMULATION.

\begin{tabular}{|c|c|c|c|c|}
\hline Simulator & Origin & $\begin{array}{l}\text { Integration } \\
\text { method }\end{array}$ & $\begin{array}{c}\text { Inter- } \\
\text { polation } \\
\end{array}$ & $\begin{array}{l}\text { Implementation } \\
\text { details }\end{array}$ \\
\hline EMTP by BPA $^{a}$ & 1968 & $\mathrm{TR}$ & - & {$[1],[2],[22]$} \\
\hline NETOMAC & 1973 & $\mathrm{TR}$ and $\mathrm{BE}$ & linear & [4], [23]-[25] \\
\hline PSCAD (EMTDC) & $1970 \mathrm{~s}$ & $\mathrm{TR}$ & linear & {$[4],[17],[26]-[31]$} \\
\hline ATP & 1984 & TR & - & {$[32],[33]$} \\
\hline EMTP by DCG $^{b}$ & 1987 & $\mathrm{TR}$ and $\mathrm{BE}$ & - & [34]-[36] \\
\hline EMTP-RV & 2003 & TR and BE & - & {$[37]-[42]$} \\
\hline XTAP & 2006 & 2s-DIRK & - & [43]-[45] \\
\hline
\end{tabular}

${ }^{a}$ Bonneville Power Administration, ${ }^{b}$ EMTP Development Coordination Group

switch events. Especially in power electronic circuits, the step size required to compensate for this loss of accuracy might be smaller than anticipated.

To assess whether it is necessary to reduce the step size and sacrifice simulation speed, insight into the behavior of approximation errors is essential. However, there is currently no comprehensive analysis of approximation errors available in the field of electromagnetic transient simulation.

Therefore, the purpose of this paper is to assess the extent to which the numerical accuracy of existing tools is affected at switch events by providing asymptotic estimates for the relation between approximation errors and the step size. In addition, this paper investigates how accuracy can be improved without losing computational performance. A benchmark method is proposed in which several improvements are incorporated to reduce the impact of switch events on the numerical accuracy. The method uses the second-order method TR-BDF2 for numerical integration between switch events [20], [21]. The $L$-stability of TR-BDF2 prevents numerical oscillations after switch events. Furthermore, quadratic interpolation is employed for event localization to match the second-order accuracy of TR-BDF2. The circuit variables are properly reinitialized after every switch event by solving the circuit statically.

Several types of errors occur in electromagnetic transient simulation [5], [13]. This paper focuses on numerical truncation errors resulting from approximating the provided continuous-time model in discrete steps. For a solution method that works properly, truncation errors converge to zero if the step size goes to zero. The paper does not deal with rounding errors resulting from using floating-point arithmetic, nor with modeling errors originating from representing the real system mathematically with a continuous-time model. 
Section II discusses the accuracy of common numerical integration methods applied to approximate the solution between switch events. Section III discusses the main causes of additional approximation errors generated at switch events and estimates the asymptotic rate at which these errors decrease for smaller steps. The benchmark method with improved asymptotic rates is proposed in Section IV. For this method, the trade-off between accuracy and the number of linear systems to be solved is compared with EMTP-RV and PSCAD for elementary circuit examples in Section V.

\section{ACCuracy of Numerical Integration Between SWITCH EVENTS}

\section{A. Circuit Equations in DAE Form}

Typical electromagnetic transient simulation tools formulate the circuit equations with an automated technique such as nodal analysis [1] or modified augmented nodal analysis [37]. With conventional lumped-circuit elements, this leads to a system of differential and algebraic equations (DAEs) [46], [47]. Each formulation technique follows a different approach to eliminate and retain unknown circuit variables. Nevertheless, as variable elimination does not affect circuit behavior, the resulting DAEs are still equivalent in the sense that they define the same solution for the output variables. Therefore, the analysis of approximation errors resulting from numerical time-discretization can be performed independently of the used formulation technique, assuming that the formulation technique does not use symbolic integration or differentiation.

In this paper, the circuit equations and the continuous-time part of the control system equations are analyzed with the common DAE form

$$
\begin{aligned}
& \dot{x}=f(x, y, t), \\
& 0=g(x, y, t),
\end{aligned}
$$

where $x$ is the vector of dynamic (differentiated) variables, and $y$ is the vector of algebraic (non-differentiated) variables [47][49]. Discrete-time control is considered separately from the continuous-time model. The equations take form (1) between control events, and some equations can be modified at control events. The operation of a switch is in essence also a control event. Similarly, the monitoring of components with piecewise-defined characteristics such as idealized diodes is also considered separately, but the system of equations will take form (1) as long as the active segment of each piecewisedefined characteristic does not change. Therefore, it may be assumed that $f$ and $g$ are sufficiently smooth between events so that the conventional literature on accuracy analysis of numerical methods for DAEs [48], [49] applies there.

For the sake of brevity, transmission line models, which are typically non-lumped models, are not considered. However, they usually decouple the circuit into multiple subcircuits, which can be solved independently as DAEs during time intervals smaller than the minimum propagation delay [1], [4].

\section{B. Local Error}

Some numerical integration methods for solving (1) are listed in Table II with their accuracy and stability properties.
TABLE II

\begin{tabular}{|c|c|c|c|c|c|c|}
\hline \multirow[b]{2}{*}{ Method } & \multirow[b]{2}{*}{ Order } & \multicolumn{2}{|c|}{$\begin{array}{l}\text { Index-1 } \\
\text { local error }^{\mathrm{a}}\end{array}$} & \multicolumn{2}{|c|}{$\begin{array}{l}\text { Index-2 } \\
\text { local error }^{\mathrm{a}}\end{array}$} & \multirow[b]{2}{*}{ Stability } \\
\hline & & in $x$ & in $y$ & in $x$ & in $y$ & \\
\hline Backward Euler & 1 & $O\left(h^{2}\right)$ & $O\left(h^{2}\right)$ & $O\left(h^{2}\right)$ & $O(h)$ & $L$-stable \\
\hline Trapezoidal method & 2 & $O\left(h^{3}\right)$ & $O\left(h^{3}\right)$ & $O\left(h^{3}\right)$ & $O\left(h^{2}\right)$ & $A$-stable \\
\hline 2s-DIRK [43], [51] & 2 & $O\left(h^{3}\right)$ & $O\left(h^{3}\right)$ & $O\left(h^{3}\right)$ & $O(h)$ & $L$-stable \\
\hline TR-BDF2 [20], [21] & 2 & $O\left(h^{3}\right)$ & $O\left(h^{3}\right)$ & $O\left(h^{3}\right)$ & $O\left(h^{2}\right)$ & $L$-stable \\
\hline
\end{tabular}

ACCURACY AND STABILITY OF (SELECTED) INTEGRATION METHODS.

${ }^{a}$ Assuming a fixed step size and exact starting values.

The local error is the error introduced in a single step, assuming exact values in earlier steps [50]. In the case of ordinary differential equations (ODEs), the order of a method quantifies the rate at which the local error reduces when taking smaller steps. On the condition that the equations are sufficiently smooth, the local error satisfies $x\left(t_{k}\right)-x_{k}=O\left(h^{p+1}\right)$ as $h \rightarrow 0$ (in asymptotic notation or "big O" notation), where $p$ is the order of the method, $h$ is the step size, $x\left(t_{k}\right)$ is the exact solution, and $x_{k}$ is the numerical solution computed assuming exact values in previous steps [50].

Error estimates for ODEs are not always valid for DAEs. Nevertheless, for the methods of Table II, the estimates remain valid for both $x$ and $y$ if (1b) uniquely determines $y$ for given $x$ and $t$ [48], [49]. DAEs with this property are said to have index one [47]-[49]. However, for many practical circuits, the DAE index is two [52], and $0=g(x, y, t)$ is not uniquely solvable on its own. In that case, some initial values of $x$ cannot be freely chosen because $g$ imposes a constraint on $x$. This happens, for instance, when two capacitors are connected in parallel, when two inductors are connected in series, or, as in Fig. 1, when a capacitor is connected in parallel with a voltage source.

An index-two DAE can often be transformed to an indexone DAE using index reduction techniques [48], [49]. Such techniques involve identification, manipulation, and symbolic differentiation of equations that impose a constraint on $x$. If an integration method is directly applied to an index-two DAE, the differentiations required for index reduction are effectively carried out numerically instead of symbolically. Error estimates for numerical integration are then generally no longer valid. The methods of Table II work well in the sense that the order of accuracy for $x$ is also valid in the index-two case. However, some variables in $y$ can suffer from a reduced order of accuracy [48], [49]. The results of Fig. 2 are obtained after applying the methods of Table II to the index-two circuit of Fig. 1 for one step. The figure shows the local error in the variables $i_{L}$ and $i_{C}$ as a function of the step size. The ODE order of accuracy associated with the methods remains valid for the dynamic variable $i_{L}$. For the algebraic variable $i_{C}$, however, order reduction occurs because numerical differentiation is applied, from $O\left(h^{2}\right)$ to $O(h)$ for backward Euler, from $O\left(h^{3}\right)$ to $O\left(h^{2}\right)$ for the trapezoidal method and for TR-BDF2, and from $O\left(h^{3}\right)$ to $O(h)$ for 2sDIRK. 


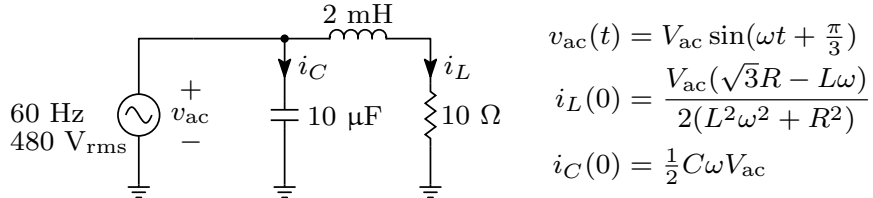

Fig. 1. Circuit with a capacitor connected in parallel with a sinusoidal voltage source. For such circuits, the formulation of the circuit equation typically leads to an index-two DAE. The inductor current $i_{L}(t)$ is initialized in periodic steady-state. The initial value of the capacitor voltage cannot be freely chosen. Computing the capacitor current $i_{C}(t)$ requires differentiation of $v_{\mathrm{ac}}(t)$.
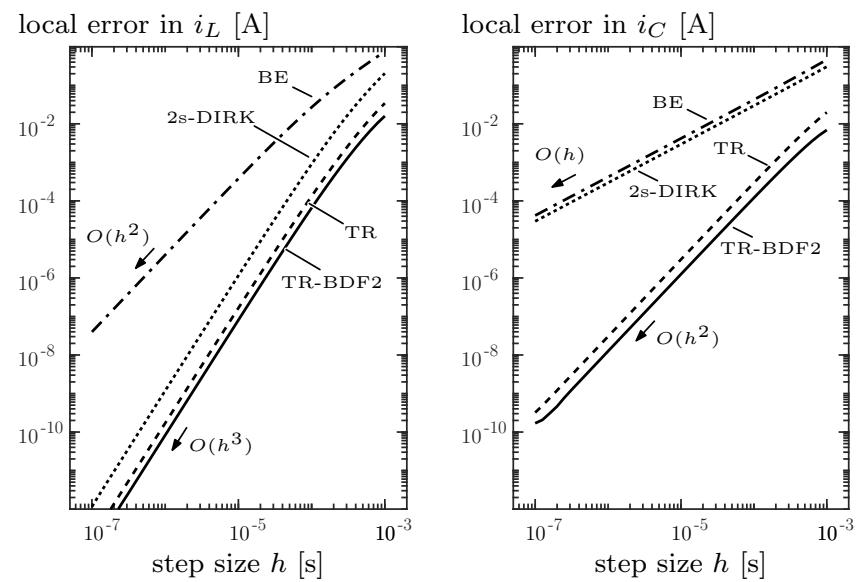

Fig. 2. Local error in the variables $i_{L}$ and $i_{C}$ as a function of the step size $h$, obtained after simulating the circuit of Fig. 1 for one step with the integration methods of Table II.

\section{Numerical Stability}

Stability properties of an integration method indicate how the step size must be restricted to preserve stability of the exact solution in the numerical solution. A-stable methods preserve stability for any step size when applied to a linear ODE of form $\dot{x}=A x$, where $A$ is a matrix [49, Definition 3.3]. Therefore, $A$-stable methods also preserve the stability of the scalar test equation $\dot{x}=\lambda x$ in which $\lambda$ is any complex or real eigenvalue of $A$. The methods of Table II are all $A$-stable. Without $A$ stability, the step size required for stability in stiff simulation problems can be unreasonably small compared to the step size required to keep the local error within desired bounds.

An $A$-stable method is also $L$-stable if a single-step solution of $\dot{x}=\lambda x$ starting at $x \neq 0$ approaches zero as $|h \lambda|$ approaches infinity [49, Definition 3.7]. As a result, such methods suppress very fast transients almost instantly in one step if the step size is sufficiently large. All methods in Table II except the trapezoidal method are $L$-stable. Although the trapezoidal method preserves stability for any step size, fast transients are not always damped as quick as in the exact solution if the step size is large relative to the time-scale of the transients. The numerical solution then often exhibits the phenomenon of slowly decaying numerical oscillations [12], [18], [49].

Numerical oscillations are a particular threat for power electronic circuits, because small on-resistances and large offresistances often cause quickly damped switching transients for which the involved time constants are much smaller than

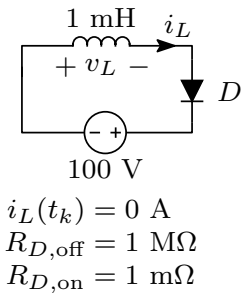

(a)

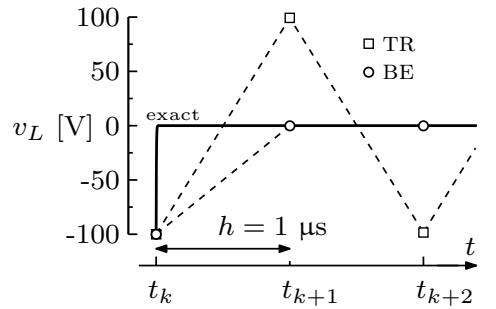

(b)

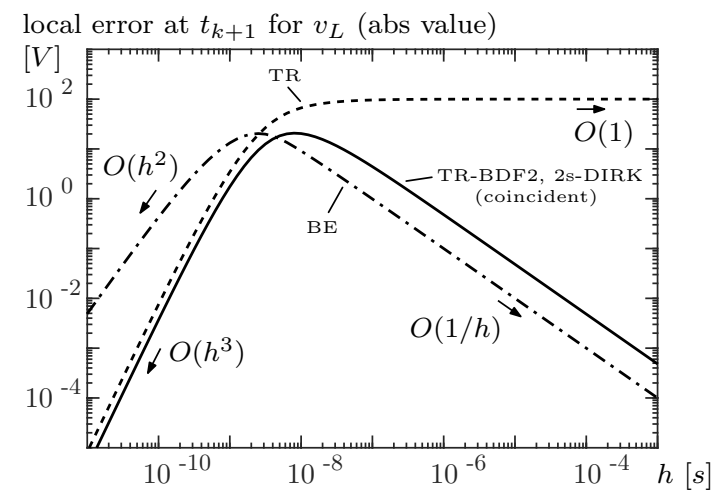

(c)

Fig. 3. Diode circuit exactly after turn-off ( $R_{\text {off }}$ is active) (a). Two-step simulation of $v_{L}$ with backward Euler (BE) and the trapezoidal method (TR) with $h=1 \mu \mathrm{s} \mathrm{(b).} \mathrm{Local} \mathrm{error} \mathrm{in} v_{L}$ at $t_{k+1}$ as a function of $h$ for BE, TR and TR-BDF2 (logarithmic scales) (c).

practical step sizes. For the circuit in Fig. 3a, for instance, the simulation in Fig. $3 \mathrm{~b}$ with the trapezoidal method exhibits numerical oscillations with $h=1 \mu \mathrm{s}$. The $L$-stable backward Euler method damps the turn-off transient almost instantly. For both methods, the local error in the first step is plotted as a function of the step size in Fig. 3c. This figure shows that the local error of the trapezoidal method is actually $O(1)$ as $h \rightarrow+\infty$ instead of $O\left(h^{3}\right)$ as $h \rightarrow 0$. With backward Euler, the error is $O(1 / h)$ as $h \rightarrow+\infty$ instead of $O\left(h^{2}\right)$ as $h \rightarrow 0$. This leads to the counter-intuitive situation in which the local error decreases as $h$ increases, on the condition that $h$ is sufficiently large.

The $O(1)$ behavior of the trapezoidal method and the $O(1 / h)$ behavior of the backward Euler method confirm that the asymptotic error rate estimates of Table II are only valid if $h$ is sufficiently small relative to the time scale of the considered variable. Therefore, strictly speaking, numerical oscillations with the trapezoidal method are not caused by the switch event itself, but by the attempt to simulate a very fast transient after switching with a too large step size. With an $L$-stable method, there are two options if a fast transient is simulated: either select $h$ sufficiently small to track the transient accurately, or select $h$ sufficiently large so that the transient is damped without accurate tracking.

The definitions of $A$-stability and $L$-stability apply only to linear time-invariant systems [49]. As switched circuits are subject to nonlinear dynamic behavior, $A$-stability does not imply that stability is preserved for any step size. To capture global dynamic behavior accurately, an appropriate step size is required and each switch event must be processed one by 
one with sufficient accuracy. Nevertheless, $L$-stable methods remain meaningful to prevent extremely small step sizes by damping very fast transients locally between switch events.

\section{Approximation ERrors AT Switch EVEnTS}

\section{A. Interpolation for Event Localization}

One cause of reduced accuracy at switch events is the approximation error made when switch events do not occur exactly at the beginning or end of a time step. EMTP, ATP, EMTP-RV, and XTAP, for instance, execute switch actions only at the end of a time step [25], [38], [42], [43]. This results in an error of $O(h)$ in variables directly affected by switch actions. Consequently, a sufficiently small step size is required to account for the lack of synchronization with events. To prevent this, several methods, including those of NETOMAC and PSCAD, employ linear interpolation to rollback the solution to the moment of switching before executing the corresponding switch actions [4], [14], [17], [19], [24], [25], [29]-[31], [53]-[57]. However, as the approximation error of linear interpolation is $O\left(h^{2}\right)$, the local $O\left(h^{3}\right)$ accuracy of the trapezoidal method is still not matched in the respective simulation steps. Linear interpolation is usually also used to resynchronize with the original time grid in subsequent steps.

\section{B. Reinitialization After Switching}

If the solution is discontinuous at a switch event, for instance after hard switching, a reinitialization is required before resuming the simulation [15]. The dynamic variables are always continuous if impulsive behavior is forbidden. Therefore, only $y$ requires reinitialization. Reinitialization with a local error up to machine precision can be accomplished by solving the circuit solution statically with $x$ fixed [15]. For index-one DAEs, this is equivalent to solving the system $0=g(x, y, t)$ for $y$. For index-two DAEs, index reduction is needed before this system can be solved uniquely.

A disadvantage is that two solvers are required in the solution procedure: one for the implicit stages of the integration method and one for reinitialization. However, the system for reinitialization can be solved more efficiently because the known variables $x$ partition the system in small subproblems [15], [58]. In [19], a reinitialization technique is presented that does not require an additional solver. However, this method is only first-order accurate because it uses backward Euler and linear extrapolation.

In PSCAD, reinitialization is accomplished using the trapezoidal method and linear interpolation [4], [30]. This method introduces an $O(h)$ error as illustrated next. Starting from $t_{k}$, a trapezoidal step is performed by solving

$$
\begin{aligned}
x_{k+1} & =m_{k+1}+\frac{h}{2} f_{1}\left(x_{k+1}, y_{k+1}, t_{k+1}\right), \\
0 & =g_{1}\left(x_{k+1}, y_{k+1}, t_{k+1}\right),
\end{aligned}
$$

where $m_{k+1}=x_{k}+\frac{h}{2} f_{1}\left(x_{k}, y_{k}, t_{k}\right)$ is the vector of history terms. If an event occurs at $t_{\alpha}$ between $t_{k}$ and $t_{k+1}$, both the solution and history terms are interpolated to the event location. Assuming exact previous steps, the resulting values $x_{\alpha}, y_{\alpha}$, and $m_{\alpha}$ have an $O\left(h^{2}\right)$ error due to the use of linear interpolation. After the interpolation, $f_{1}$ and $g_{1}$ are modified to $f_{2}$ and $g_{2}$ according to the associated switch actions. PSCAD then reinitializes the solution to $x_{\alpha}^{*}$ and $y_{\alpha}^{*}$ with a trapezoidal step from $t_{\alpha}-h$ to $t_{\alpha}$ using $m_{\alpha}$ by solving

$$
\begin{aligned}
x_{\alpha}^{*} & =m_{\alpha}+\frac{h}{2} f_{2}\left(x_{\alpha}^{*}, y_{\alpha}^{*}, t_{\alpha}\right), \\
0 & =g_{2}\left(x_{\alpha}^{*}, y_{\alpha}^{*}, t_{\alpha}\right) .
\end{aligned}
$$

As $x$ is assumed to be continuous, another approximation for the exact solution of $x$ at $t_{\alpha}$ is

$$
\tilde{x}_{\alpha}=m_{\alpha}+\frac{h}{2} f_{1}\left(x_{\alpha}, y_{\alpha}, t_{\alpha}\right)=x\left(t_{\alpha}\right)+O\left(h^{2}\right) .
$$

By taking the difference between (4) and (3a), it is verified that the introduced error is $O(h)$ unless $f_{1}=f_{2}$. Because this error occurs in $x$, propagation to subsequent steps is inevitable.

\section{Suppression of Numerical Oscillations}

Another type of approximation error is made by techniques employed to suppress unphysical numerical oscillations caused by the trapezoidal method at switching transients. Without provisions, artificial snubber circuits must be added to enforce augmented damping [4], [5], [12], [13], [18], [22], resulting in additional modeling errors.

An effective approach is to use an $L$-stable method. NETOMAC, later versions of DCG-EMTP, and EMTP-RV (with the "Trapezoidal and Backward Euler" integration option) perform one or two backward Euler steps of size $h / 2$ after switch events [4], [18], [24], [34], [41]. However, as backward Euler has order one, $O\left(h^{3}\right)$ accuracy is lost for all variables. PSCAD uses a half-way linearly interpolated trapezoidal step to suppress oscillations [4], [17], [31]. This method is also only first-order accurate due to the use of linear interpolation. The second-order 2 s-DIRK method of XTAP already suppresses numerical oscillations on its own. Therefore, $O\left(h^{3}\right)$ accuracy is preserved for variables unaffected by asynchronous events.

\section{Interpolation of Suppressed Variables}

While the error of linear interpolation is $O\left(h^{2}\right)$ for variables tracked with a sufficiently small $h$, an additional approximation error occurs if interpolation is performed on a variable of which fast switching transients are suppressed with an $L$ stable method. This approximation error is caused because the damping capability of $L$-stable methods is generally not preserved in steps with interpolation [59]. For example, with the backward Euler method applied to $\dot{x}=\lambda x$ with $\operatorname{Re}(\lambda)<0$, linear interpolation at position $t_{k}+\alpha h$ gives

$$
x^{*}=(1-\alpha) x_{k}+\alpha x_{k+1}=(1-\alpha) x_{k}+\alpha \frac{1}{1-h \lambda} x_{k},
$$

with $0<\alpha \leq 1$. In the limit for $h \rightarrow+\infty, x_{k+1}$ approaches the exact solution zero, but $x^{*}$ approaches $(1-\alpha) x_{k}$, which is only zero for $\alpha=1$. Hence, the error is $O(1)$ as $h \rightarrow+\infty$ for $0<\alpha<1$. Especially in the first step of a switching transient, this error can be significant if $x_{k}$ is not yet sufficiently damped.

A similar derivation can be used to show that the linearly interpolated trapezoidal method is only $L$-stable for $\alpha=1 / 2$. This explains why the method used to suppress numerical oscillations in PSCAD is effective. Nevertheless, $O(1)$ errors 
TABLE III

SUMMARY OF EXPECTED LOCAL APPROXIMATION ERRORS

\begin{tabular}{|c|c|c|c|}
\hline Origin & EMTP-RV & PSCAD & Benchmark \\
\hline Step without events ${ }^{\mathrm{a}}$ & $O\left(h^{3}\right)$ & $O\left(h^{3}\right)$ & $O\left(h^{3}\right)$ \\
\hline Unsychronized event & $O(h)$ & - & - \\
\hline \multicolumn{4}{|c|}{ Interpolation / resynchronization } \\
\hline tracked variables & - & $O\left(h^{2}\right)$ & $O\left(h^{3}\right)$ \\
\hline suppressed variables & - & $O(1)$ & $O(1)$ \\
\hline Reinitialization & - & $O(h)$ & $O(\epsilon)^{\mathrm{b}}$ \\
\hline \multicolumn{4}{|l|}{ Suppression of oscillations } \\
\hline tracked variables & $O\left(h^{2}\right)$ & $O\left(h^{2}\right)$ & $O\left(h^{3}\right)$ \\
\hline suppressed variables & $O\left(h^{-1}\right)$ & $O\left(h^{-1}\right)$ & $O\left(h^{-1}\right)$ \\
\hline
\end{tabular}

${ }^{\mathrm{a}}$ Assuming that the variable is not suffering from index-2 order reduction.

${ }^{\mathrm{b}} \mathrm{Up}$ to machine precision.

also occur because resynchronization with the original time grid is generally performed with $\alpha \neq 1 / 2$.

In the numerical examples of this paper, the discussed $O(1)$ errors do not propagate significantly because they are damped in subsequent time steps. However, specific cases involving diode bridge commutation or short moments of discontinuous conduction may lead to propagated errors due to inaccurate localization of events in a step with $O(1)$ accuracy [59].

\section{E. Error propagation}

Table III summarizes the local approximation errors expected in EMTP-RV and PSCAD. It is not straightforward to predict the effect of local errors on the global error obtained after simulating a time interval $\left[0, t_{\text {end }}\right]$, because error propagation is governed by system dynamics. Assuming that propagated errors are not magnified by system dynamics and that the error introduced locally in every step satisfies $\left|e_{k}^{L}\right| \leq M h^{q}$ for a constant $M$, a rough asymptotic estimate for the global error is $O\left(n_{s} M h^{q}\right)=O\left(h^{q-1}\right)$ using $n_{s}=t_{\text {end }} / h$.

To assess the overall accuracy of a waveform during the simulation interval, the relative rms error is used in this paper. For a variable $x$, this error is defined as

$$
e_{\mathrm{rms}}=\frac{1}{x_{\mathrm{rms}}} \sqrt{\frac{1}{n_{s}} \sum_{k=1}^{n_{s}}\left(x\left(t_{k}\right)-x_{k}\right)^{2}},
$$

where $x(t)$ is the exact solution and $x_{\mathrm{rms}}$ the rms value of the exact solution. Again assuming $\left|e_{k}^{L}\right| \leq M h^{q}$ at every step without magnified propagation, we have

$$
e_{\mathrm{rms}} \leq \sqrt{\frac{1}{n_{s}} \sum_{k=1}^{n_{s}}\left(k M h^{q}\right)^{2}}=O\left(h^{q-1}\right),
$$

using $\sum_{k=1}^{n_{s}} k^{2}=O\left(n_{s}^{3}\right)$. If the rms error is dominated by an $O\left(h^{q}\right)$ error introduced at a finite number of events,

$$
e_{\mathrm{rms}} \leq \sqrt{\frac{1}{n_{s}} \sum_{i=1}^{n_{e}} \frac{t_{i+1}-t_{i}}{h}\left(i M h^{q}\right)^{2}}=O\left(h^{q}\right)
$$

is expected, where $n_{e}$ is the number of events, $t_{n_{e}+1}=t_{\mathrm{end}}$, and $t_{i}$ with $i \leq n_{e}$ is the time instant at which each event occurs. If event errors do not propagate to subsequent steps, or if they are damped to an insignificant level in a finite number of steps, this rate estimate can be further refined to

$$
e_{\mathrm{rms}} \leq \sqrt{\frac{1}{n_{s}} \sum_{i=1}^{n_{e}} C_{i} M^{2} h^{2 q}}=O\left(h^{q+\frac{1}{2}}\right),
$$

in which $C_{i}$ is a constant denoting for how many steps the error remains significant.

\section{F. Other Sources of Errors not Discussed in Detail}

1) Control System Delays: In some tools, artificial interfacing delays are introduced in the control system model [12][14], [35], [39]. While the error of a one-step delayed smooth control signal is $O(h)$, the error can be more severe if switches are controlled with non-smooth control laws.

2) Simultaneous Switch Actions: The operation of one switch often induces changes in other switches [15]. To prevent additional errors due to delayed switch actions, a valid switch configuration must be found without advancing in time [15], [16], [19]. EMTP-RV, with the "Simultaneous Switching" option enabled, rejects exploratory steps until a valid switch configuration is found [42]. PSCAD repeats reinitialization until the configuration is valid [4], [30].

3) Transmission Lines: Additional analysis is required to assess the approximation errors resulting from discretizing transmission line models. This can be done by analyzing the accuracy of solving the associated convolution integrals numerically, which is $O\left(h^{3}\right)$ when the trapezoidal rule is used and when the input signals are smooth. Further research is required to investigate how the accuracy of existing methods behaves if the input signals are non-smooth due to switching.

\section{BENCHMARK METHOD}

The proposed benchmark method is based on the integration method TR-BDF2 [20], [21]. TR-BDF2 combines an internal trapezoidal step and an internal BDF2 step into a single-step method. The resulting method has order two, is $A$-stable, and inherits the $L$-stability of BDF2. The formula is given by

$$
\begin{aligned}
x_{\gamma} & =x_{k}+\gamma \frac{h}{2} f\left(x_{k}, y_{k}, t_{k}\right)+\gamma \frac{h}{2} f\left(x_{\gamma}, y_{\gamma}, t_{\gamma}\right) \\
0 & =g\left(x_{\gamma}, y_{\gamma}, t_{\gamma}\right), \\
x_{k+1} & =(1-\eta) x_{k}+\eta x_{\gamma}+\gamma \frac{h}{2} f\left(x_{k+1}, y_{k+1}, t_{k+1}\right), \\
0 & =g\left(x_{k+1}, y_{k+1}, t_{k+1}\right),
\end{aligned}
$$

where $\gamma=2-\sqrt{2}$ and $\eta=\frac{1+\sqrt{2}}{2}$. The internal trapezoidal step has size $\gamma h$, the subsequent internal BDF2 step has size $(1-\gamma) h$. The factor in front of $f$ is $\gamma \frac{h}{2}$ for both implicit stages, allowing to use the same $L U$ factorization to solve each stage if the corresponding system of equations is linear. In the nonlinear case, Jacobian factorizations can be reused with the simplified Newton iteration approach [20], [21].

The intermediate solution point of the internal stage of TRBDF2 is also second-order accurate, and can therefore be used as a point for quadratic Lagrange interpolation. The resulting quadratic interpolation function is

$$
\begin{aligned}
& \tilde{x}_{k+1}(\alpha)=p_{1}(\alpha) x_{k}+p_{2}(\alpha) x_{\gamma}+p_{3}(\alpha) x_{k+1}, \\
& \tilde{y}_{k+1}(\alpha)=p_{1}(\alpha) y_{k}+p_{2}(\alpha) y_{\gamma}+p_{3}(\alpha) y_{k+1},
\end{aligned}
$$


with

$$
\begin{aligned}
& p_{1}(\alpha)=\gamma^{-1}(\alpha-\gamma)(\alpha-1) \\
& p_{2}(\alpha)=\frac{\alpha(\alpha-1)}{\gamma(\gamma-1)}, \quad \text { and } \quad p_{3}(\alpha)=\frac{\alpha(\alpha-\gamma)}{(1-\gamma)} .
\end{aligned}
$$

As the error introduced by quadratic interpolation is $O\left(h^{3}\right)$, the second-order accuracy of the integration method is preserved in both $x$ and $y$.

The quadratic interpolant is used to locate the exact moment of switching if a sign change is detected in one of the guard variables. Quadratic interpolation is also used for resynchronization with the original time grid. Reinitialization after hard switching is achieved by solving $g(x, y, t)=0$ statically. The reinitialization is repeated until a valid switch configuration is found to resolve simultaneous switch actions. In steps with switch events, $O\left(h^{3}\right)$ accuracy is preserved in variables for which the step size is selected sufficiently small.

The expected local approximation errors are compared with EMTP-RV and PSCAD in Table III. Numerical oscillations are automatically suppressed with an $O(1 / h)$ suppression error as illustrated in Fig. 3c. As $L$-stability is not preserved by interpolation, local $O(1)$ errors are still possible if a suppressed variable is interpolated. In [59], a quadratic interpolation technique is presented that avoids $O(1)$ errors.

The method TR-BDF2 is similar to 2s-DIRK. Both methods are $L$-stable and involve two stages [20], [21], [43], [51]. Two important differences are that 2s-DIRK suffers more from order reduction with index-two problems, as illustrated in Fig. 2, and that the intermediate solution point of the internal stage of 2s-DIRK is only first-order accurate. As a result, quadratic Lagrange interpolation cannot be applied using the intermediate solution point.

\section{NUMERICAL EXAMPLES}

As inaccuracies are often masked by the complexity of a system, the numerical examples are deliberately kept simple. Each example is simulated with EMTP-RV 3.3.1 (demo) and PSCAD 4.6.0. The accurate reference solution is computed with the simulation tool PLECS 3.7.2 [60], using the variablestep solver ode 45 with an absolute and relative tolerance of $10^{-9}$, a maximum step size of $100 \mathrm{~ns}$, and all other settings put to default. The benchmark method is implemented in Matlab.

\section{A. Thyristor Controlled Reactor}

The first example is a single-phase thyristor controlled reactor (TCR), shown in Fig. 4. The TCR is controlled with a fixed firing angle. The circuit is simulated for $50 \mathrm{~ms}$ with PSCAD and EMTP-RV using $h=50 \mu \mathrm{s}$, and with the benchmark method using $h=500 \mu$ s. The reference solution for the inductor current $i_{L}$ is displayed with the simulation errors for each tool in Fig. 5. With PSCAD and EMTP-RV, the most significant error is introduced when the thyristors turn on. This error is canceled out by a local error of opposite sign when the thyristors turn off. The relative rms error of the signal $i_{L}$ is $2.23 \times 10^{-2}$ for PSCAD, $1.30 \times 10^{-2}$ for EMTP-RV, and $1.35 \times 10^{-3}$ for the benchmark method. Although PSCAD uses interpolation and EMTP-RV does not, both tools produce

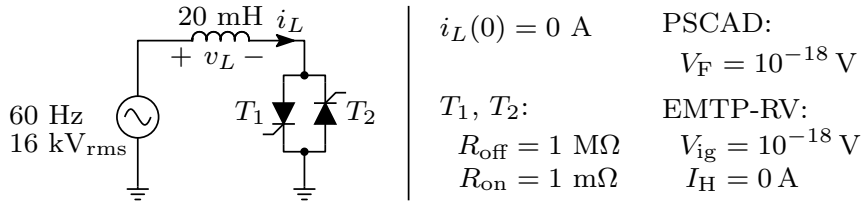

Fig. 4. Single-phase TCR circuit controlled with firing angle $\alpha_{\text {fire }}=120^{\circ}$.
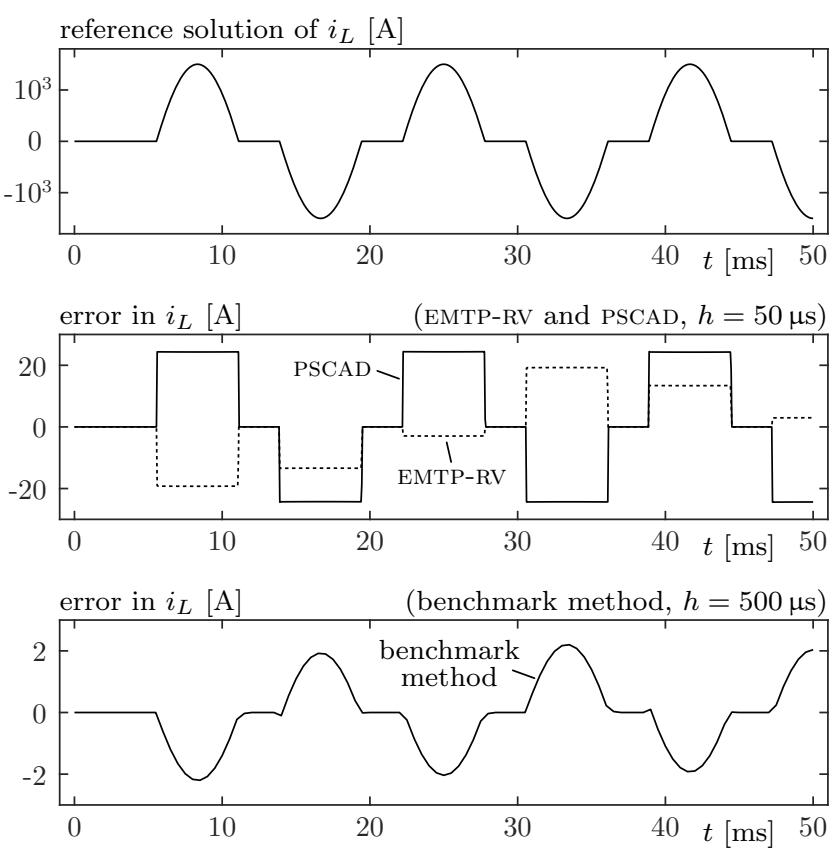

Fig. 5. Reference solution of $i_{L}$ for the circuit in Fig. 4 and simulation error of EMTP-RV, PSCAD, and the benchmark method.

an error of the same order of magnitude. The benchmark method is one order of magnitude more accurate with a ten times greater step size, indicating that the advantage of improved accuracy outweighs the disadvantage of increased complexity of the method.

Next, the trade-off between accuracy and computational work is investigated. In order to obtain an implementationindependent comparison, the computational work is estimated in terms of the number of linear systems to be solved because it is assumed that most work is spent there for large circuits. Although $L U$-factorizations can be reused for subsequent steps between switch events, the number of $L U$-factorization computations is expected to be the same for each of the compared methods, because the number of times a $L U$-factorization is needed does not change with the step size.

EMTP-RV solves one system for each TR or BE step and for each rejected exploratory step at switch events. PSCAD solves one system for each regular step, for each reinitialization after hard switching, for each half-way linearly interpolated TR step, and for each resynchronization step. The benchmark method requires two system solves for each regular TR-BDF2 step and for each resynchronization step. Reinitializations are also counted as full system solves, although it is expected that the system for reinitialization can be solved more efficiently.

The trade-off curves for $i_{L}$ in Fig. 6 are obtained by computing the relative rms error $e_{\mathrm{rms}}$ and the number of system solves 

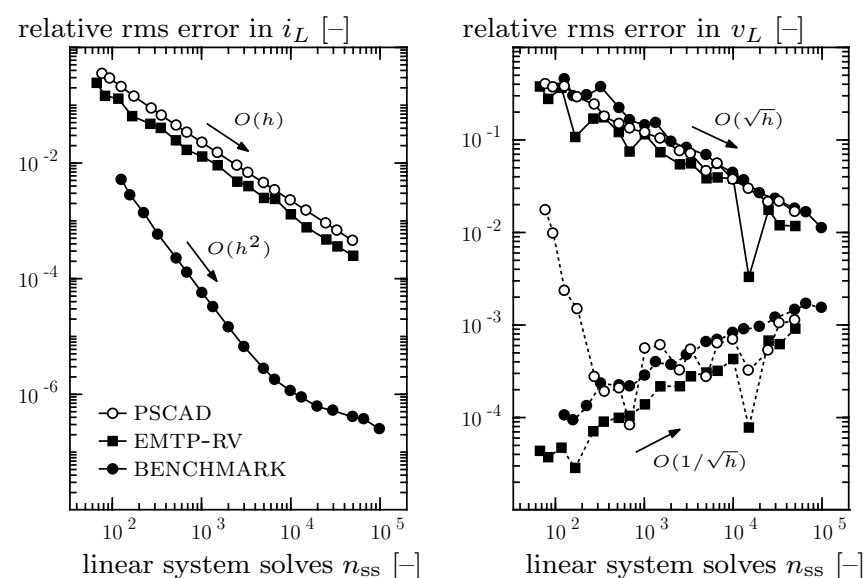

Fig. 6. Trade-off curves between the relative rms error in the variables $i_{L}$ and $v_{L}$ and the number of linear systems solved. The curves are obtained after simulating an interval of $50 \mathrm{~ms}$ with different step sizes. The dashed curves for $v_{L}$ exclude the first solution point after each switch event in the computation of the relative rms error.

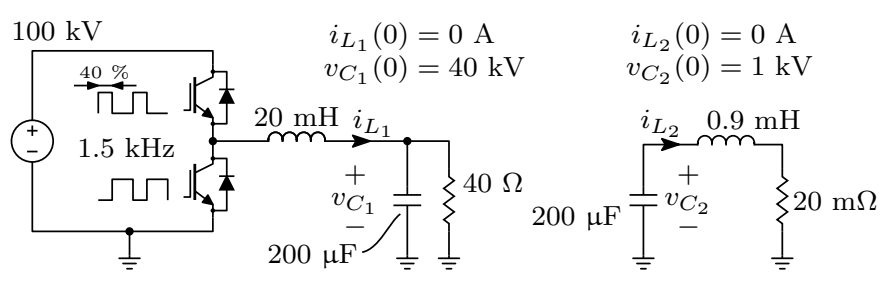

Fig. 7. Half-bridge circuit simulated simultaneously with an RLC circuit with a resonance frequency of $375.13 \mathrm{~Hz} . R_{\mathrm{on}}=1 \mathrm{~m} \Omega$ and $R_{\text {off }}=1 \mathrm{M} \Omega$ for each IGBT and for each diode.

$n_{\mathrm{ss}}$ for different step sizes. PSCAD and EMTP-RV achieve only $O\left(1 / n_{\mathrm{ss}}\right)=O(h)$ accuracy in the relative rms error. The only propagation formula of Section III-E that can explain $O(h)$ accuracy with the local error estimates of Table III is (8). For PSCAD, this indicates that the propagating $O(h)$ error introduced by the reinitialization technique at switch events is the most significant error. For EMTP-RV, the propagating $O(h)$ error is caused by unsynchronized switch events. With the benchmark method, the second-order accuracy of TRBDF2 is preserved. For a fixed amount of computational work, two to three orders of magnitude of accuracy are gained compared to PSCAD and EMTP-RV.

The trade-off curves for the variable $v_{L}$ are shown in Fig. 6 (full lines). All tools achieve $O(\sqrt{h})$ accuracy. With (9), $O(\sqrt{h})$ accuracy can be shown to originate from nonpropagating $O(1)$ local errors at switch events. For PSCAD and the benchmark method, this error is due to interpolation of the a suppressed variable at resynchronization steps. Numerical oscillations in $v_{L}$ are indeed suppressed after thyristor commutation. If the first solution point after each event is excluded in the computation of the relative rms error for $v_{L}$ (dashed lines), an $O(1 / \sqrt{h})$ trade-off curve is obtained, indicating quickly damped $O(1 / h)$ local errors at switch events.
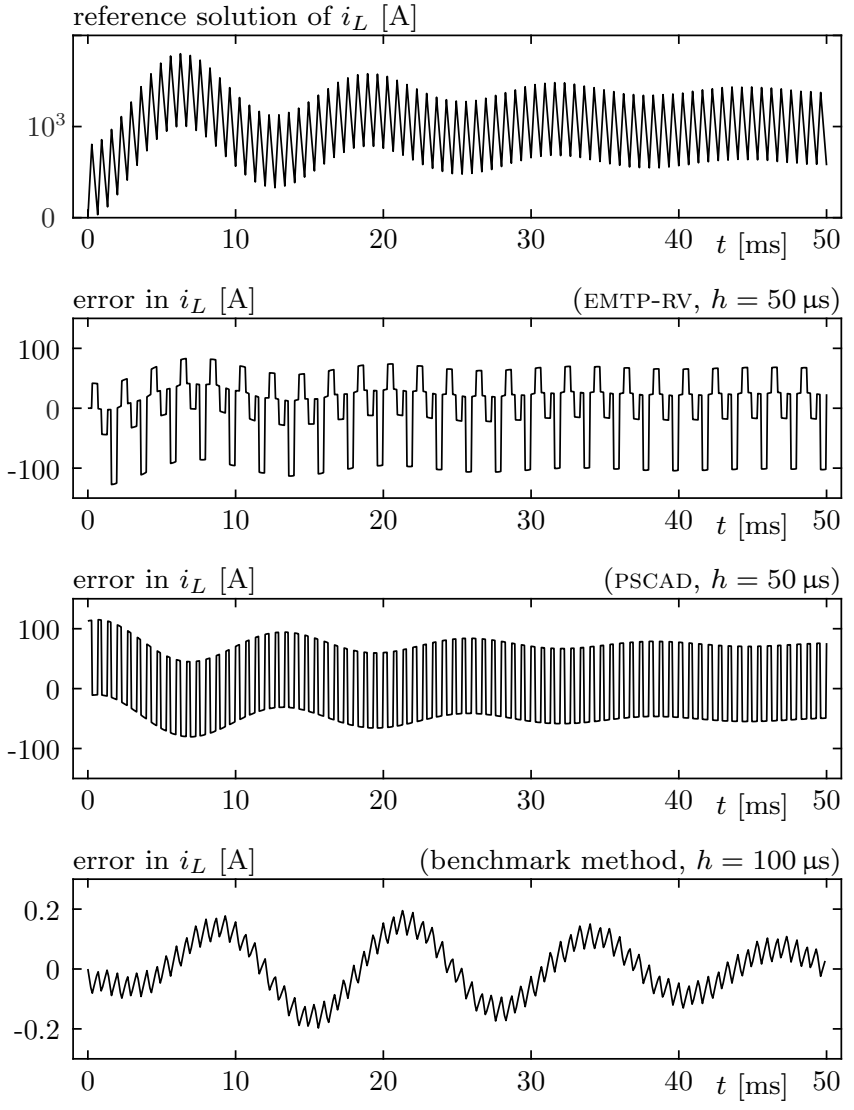

Fig. 8. Reference solution of $i_{L}$ for the circuit in Fig. 7 and simulation error of EMTP-RV, PSCAD, and the benchmark method.

\section{B. PWM-Controlled IGBT Half-Bridge}

In the example of Fig. 7, a PWM-controlled half-bridge circuit is simulated simultaneously with an RLC resonance circuit. The circuit is simulated for $50 \mathrm{~ms}$ with PSCAD and EMTP-RV using $h=50 \mu \mathrm{s}$, and with the benchmark method using $h=100 \mu$ s. In PSCAD, initial conditions for capacitors are imposed by connecting a voltage source in series. The reference solution for the inductor current $i_{L_{1}}$ is shown with the simulation errors for each tool in Fig. 8. With PSCAD and EMTP-RV, the most significant error is again introduced at switch events. The relative rms error of $i_{L_{1}}$ is $6.08 \times 10^{-2}$ for PSCAD, $5.52 \times 10^{-2}$ for EMTP-RV, and $8.60 \times 10^{-5}$ for the benchmark method. For this variable, the benchmark method is almost three orders of magnitude more accurate.

The trade-off curves for $i_{L_{1}}$ and $v_{C_{1}}$, two variables directly affected by switch actions, are shown in Fig. 9. The number of linear systems to be solved is computed in the same way as in Section V-A. One additional system solve is required at every switch event for rejected exploratory steps or reinitializations when one of the diodes goes into or out of conduction. For EMTP-RV and PSCAD, the $O(h)$ error at switch events is limiting the accuracy of both variables. A small step size and a large amount of linear system solves are required to achieve a relative rms error below $1 \times 10^{-3}$. With the benchmark method, reducing the step size quickly leads to an accuracy improvement of several orders of magnitude.

The trade-off curves for the RLC current $i_{L_{2}}$ are shown 

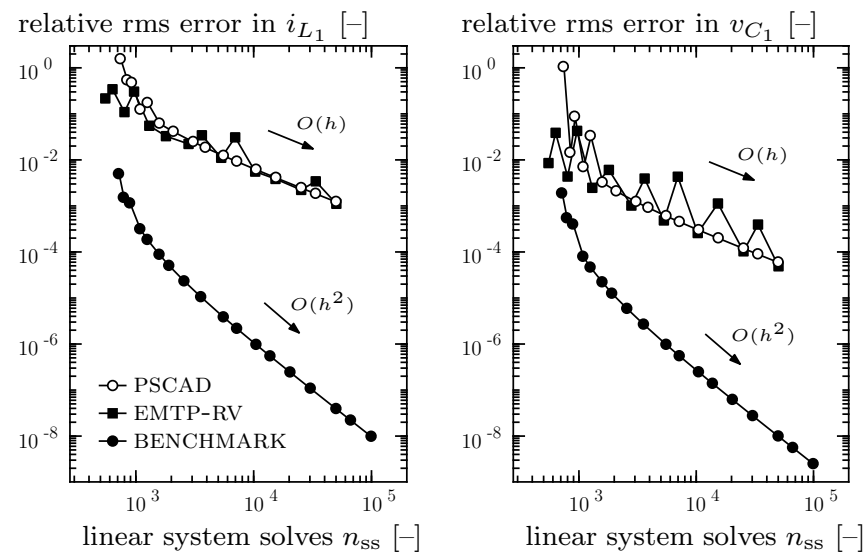

Fig. 9. Trade-off curves between the relative rms error in the variables $i_{L_{1}}$ and $v_{C_{1}}$ and the number of linear systems solved. The curves are obtained after simulating an interval of $50 \mathrm{~ms}$ with different step sizes.
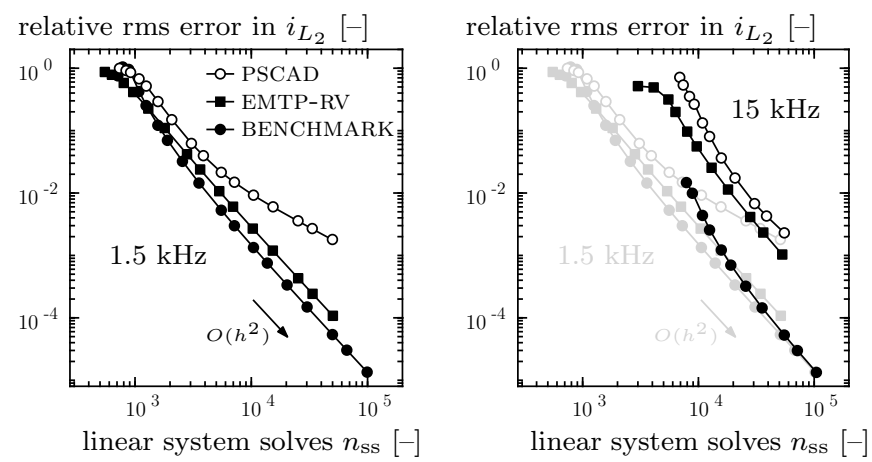

Fig. 10. Trade-off curves between the relative rms error in the RLC current $i_{L_{2}}$ and the number of linear systems solved, for a switching frequency of $1.5 \mathrm{kHz}$ and $15 \mathrm{kHz}$.

in Fig. 10 for a switching frequency of $1.5 \mathrm{kHz}$ and $15 \mathrm{kHz}$. Although the exact solution is not dependent on switch actions, the accuracy of $i_{L_{2}}$ with EMTP-RV and PSCAD reduces if the switching frequency increases. This illustrates that the use of backward Euler or linear interpolation at switch events influences the accuracy of all variables. With the benchmark method, the accuracy does not change that much.

\section{CONCLUSION}

In current tools for electromagnetic transient simulation with support for power electronics, the second-order accuracy of the trapezoidal method is not preserved at switch events. Numerical examples demonstrate that simulation variables can be up to several orders of magnitude less accurate than generally expected from a second-order method. Furthermore, the asymptotic error estimates provided in this paper indicate that the accuracy at switch events does not improve as quick as with a second-order method when the step size is reduced. Consequently, the step size required to improve accuracy often leads to an unwanted slowdown of the simulation.

Three improvements are proposed in particular: the use of a second-order $L$-stable method such as TR-BDF2 for the suppression of numerical oscillations, quadratic interpolation for event localization, and accurate reinitialization through separation of the solvers for reinitialization and integration. Results obtained using the proposed improvements show the potential to significantly improve the trade-off between accuracy and the number of linear systems to be solved. The improvements are sufficiently large to justify the added complexity of using a two-stage method instead of a onestage method, of implementing quadratic interpolation instead of linear interpolation, and of requiring a separate solver for reinitialization.

While the examples of this paper are deliberately kept simple for illustration purposes, the provided asymptotic error estimates are valid for variables in large-scale power electronic circuits as well. For large circuits, most computation time is spent in solving linear systems. Therefore, the improved tradeoff between accuracy and the number of linear systems to be solved directly translates into a reduction of computation time for the desired accuracy. Further research is required to assess the impact of the approximation errors in current tools on the results of simulation studies with large-scale power electronic circuits such as HVDC systems with modular multilevel converters. Due to the complexity of such circuits, it is often difficult to distinguish large approximation errors from normal system behavior. The analysis provided in this paper can assist in investigating the cause of suspicious behavior.

\section{REFERENCES}

[1] H. W. Dommel, "Digital computer solution of electromagnetic transients in single-and multiphase networks," IEEE Trans. Power Appar. Syst., vol. PAS-88, no. 4, pp. 388-399, Apr. 1969.

[2] H. W. Dommel and W. S. Meyer, "Computation of electromagnetic transients," Proc. IEEE, vol. 62, no. 7, pp. 983-993, Jul. 1974.

[3] J. Mahseredjian, V. Dinavahi, and J. A. Martinez, "Simulation tools for electromagnetic transients in power systems: Overview and challenges," IEEE Trans. Power Del., vol. 24, no. 3, pp. 1657-1669, Jul. 2009.

[4] N. Watson and J. Arrillaga, Power Systems Electromagnetic Transients Simulation, ser. IET power and energy series 39, A. T. Johns and D. F. Warne, Eds. London, UK: IET, 2003, vol. 39.

[5] J. A. Martinez-Velasco, Ed., Transient Analysis of Power Systems: Solution Techniques, Tools and Applications. Wiley-IEEE Press, 2015.

[6] A. Ametani, Ed., Numerical Analysis of Power System Transients and Dynamics, ser. Energy Engineering. London, UK: IET, 2015.

[7] A. M. Gole, "Electromagnetic transient simulation of power electronic equipment in power systems: challenges and solutions," 2006 IEEE Power Eng. Soc. Gen. Meet., pp. 6-11, 2006.

[8] "Modelling and simulation studies to be performed during the lifecycle of HVDC systems," CIGRE, Tech. Broch. 5632013 B4-38, 2013.

[9] O. Nayak, S. Santoso, and P. Buchanan, "Power electronics spark new simulation challenges," IEEE Comput. Appl. Power, vol. 15, no. 4, pp. 37-44, 2002.

[10] A. Beddard, M. Barnes, and R. Preece, "Comparison of detailed modeling techniques for MMC employed on VSC-HVDC schemes," IEEE Trans. Power Del., vol. 30, no. 2, pp. 579-589, Apr. 2015.

[11] B. Johnson, H. Hess, and J. A. Martinez, "Parameter determination for modeling system transients-part VII: Semiconductors," IEEE Trans. Power Del., vol. 20, no. 3, pp. 2086-2094, Jul. 2005.

[12] N. Mohan, W. P. Robbins, T. M. Undeland, and R. Nilssen, "Simulation of power electronic and motion control systems-an overview," Proc. IEEE, vol. 82, no. 8, pp. 1287-1302, 1994.

[13] A. M. Gole, A. Keri, C. Kwankpa, E. W. Gunther, H. W. Dommel, I. Hassan, J. R. Marti, J. A. Martinez, K. G. Fehrle, L. Tang, M. F. McGranaghan, O. B. Nayak, P. F. Ribeiro, R. Iravani, and R. Lasseter, "Guidelines for modeling power electronics in electric power engineering applications," IEEE Trans. Power Del., vol. 12, no. 1, pp. 505-514, 1997.

[14] A.-R. Sana, J. Mahseredjian, X. Dai-Do, and H. Dommel, "Treatment of discontinuities in time-domain simulation of switched networks," Math. Comput. Simul., vol. 38, no. 4-6, pp. 377-387, Aug. 1995. 
[15] B. De Kelper, L. A. Dessaint, K. Al-Haddad, and H. Nakra, "A comprehensive approach to fixed-step simulation of switched circuits," IEEE Trans. Power Electron., vol. 17, no. 2, pp. 216-224, Mar. 2002.

[16] J. Mahseredjian and G. Joos, "Solution methods for varying topology networks," in Proc. Power Eng. Soc. Winter Meet. IEEE, 2002, pp. 479-483.

[17] P. Kuffel, K. Kent, and G. Irwin, "The implementation and effectiveness of linear interpolation within digital simulation," Int. J. Electr. Power Energy Syst., vol. 19, no. 4, pp. 221-227, May 1997.

[18] J. R. Marti and J. Lin, "Suppression of numerical oscillations in the EMTP," IEEE Trans. Power Syst., vol. 4, no. 2, pp. 739-747, May 1989.

[19] M. Zou, J. Mahseredjian, G. Joos, B. Delourme, and L. Gérin-Lajoie, "Interpolation and reinitialization in time-domain simulation of power electronic circuits," Electr. Power Syst. Res., vol. 76, no. 8, pp. 688-694, May 2006.

[20] R. E. Bank, W. M. Coughran, W. Fichtner, E. H. Grosse, D. J. Rose, and R. K. Smith, "Transient simulation of silicon devices and circuits," IEEE Trans. Electron Devices, vol. 32, no. 4, pp. 1992-2007, 1985.

[21] M. E. Hosea and L. F. Shampine, "Analysis and implementation of TRBDF2," Appl. Numer. Math., vol. 20, pp. 21-37, 1996.

[22] H. W. Dommel, Electromagnetic Transients Program Theory Book. Bonneville Power Administration, 1986.

[23] B. Kulicke, "Digitalprogramm NETOMAC zur simulation elektromagnetischer und -magnetischer ausgleichsvorgänge in drehstromnetzen," Elektrizitätswirtschaft, vol. 78, no. 1, pp. 18-23, 1979.

[24] B. Kulicke, E. Lerch, O. Ruhle, and W. Winter, "NETOMAC - calculating, analyzing and optimizing the dynamic of electrical systems in time and frequency domain," in Proc. Int. Conf. Power Syst. Transients, Budapest, Hungary, Jun. 1999.

[25] P. Lehn, J. Rittiger, and B. Kulicke, "Comparison of the ATP version of the EMTP and the NETOMAC program for simulation of HVDC systems," IEEE Trans. Power Del., vol. 10, no. 4, pp. 2048-2053, 1995.

[26] D. A. Woodford, A. M. Gole, and R. W. Menzies, "Digital simulation of DC links and AC machines," IEEE Trans. Power Appar. Syst., vol. PAS-102, no. 6, pp. 1616-1623, Jun. 1983.

[27] D. Woodford, G. Irwin, and U. S. Gudmundsdottir, "PSCAD/EMTDC," in Numer. Anal. Power Syst. Transients Dyn. IET, Jan. 2015, ch. 4, pp. $135-167$.

[28] O. Nayak, G. Irwin, and A. Neufeld, "GUI enhances electromagnetic transients simulation tools," IEEE Comput. Appl. Power, vol. 8, no. 1, pp. 17-22, 1995.

[29] A. M. Gole, S. A. Woodford, J. E. Nordstrom, and G. D. Irwin, "A fully interpolated controls library for electromagnetic transients simulation of power electronic systems," in Proc. Int. Conf. Power Syst. Transients, Rio de Janeiro, Brazil, 2001.

[30] G. D. Irwin, D. A. Woodford, and A. Gole, "Precision simulation of PWM controllers," in Proc. Int. Conf. Power Syst. Transients, Rio de Janeiro, Brazil, 2001.

[31] EMTDC User's Guide, Manitoba HVDC Research Centre, Sep. 2015, version 4.6. [Online]. Available: https://hvdc.ca/

[32] M. Kizilcay and H. K. Hoidalen, "EMTP-ATP," in Numer. Anal. Power Syst. Transients Dyn., A. Ametani, Ed. IET, Jan. 2015, ch. 2, pp. 47-101.

[33] T. Funaki, H. Arita, A. Kan, and Y. Kashiwagi, "Handling of switching transient and GIFU switch ability on the IEE-J's power electronics benchmark circuits," in Proc. Int. Conf. Power Syst. Transients, Rio de Janeiro, Brazil, 2001.

[34] J. Lin and J. R. Marti, "Implementation of the CDA procedure in the EMTP," IEEE Trans. Power Syst., vol. 5, no. 2, pp. 394-402, May 1990.

[35] R. H. Lasseter and J. Zhou, "TACS enhancements for the electromagnetic transient program," IEEE Trans. Power Syst., vol. 9, no. 2, pp. 736-742, May 1994.

[36] S. Lefebvre and R. D. Rangel, "Modeling of power electronics devices in EMTP-TACS," in Proc. Int. Conf. Power Syst. Transients, Lisbon, Portugal, 1995.

[37] J. Mahseredjian, S. Dennetière, L. Dubé, B. Khodabakhchian, and L. Gérin-Lajoie, "On a new approach for the simulation of transients in power systems," Electr. Power Syst. Res., vol. 77, no. 11, pp. 1514-1520, Sep. 2007.

[38] J. Mahseredjian, U. Karaagac, S. Dennetière, and H. Saad, "Simulation of electromagnetic transients with EMTP-RV," in Numer. Anal. Power Syst. Transients Dyn. IET, Jan. 2015, ch. 3, pp. 103-134.
[39] J. Mahseredjian, L. Dubé, M. Zou, S. Dennetière, and G. Joos, "Simultaneous solution of control system equations in EMTP," IEEE Trans. Power Syst., vol. 21, no. 1, pp. 117-124, 2006.

[40] J. Mahseredjian and C. Dewhurst, EMTP-RV User Manual, Powersys, Jan. 2016, version 3.3 .

[41] J. Mahseredjian, EMTP-EMTPWorks simulation options, Powersys, Oct. 2014, EMTP-RV Version 3.3 [Help document].

[42] - Simultaneous switching option, Powersys, Apr. 2016, EMTP-RV Version 3.3 [Help document].

[43] T. Noda, K. Takenaka, and T. Inoue, "Numerical integration by the 2-stage diagonally implicit RungeKutta method for electromagnetic transient simulations," IEEE Trans. Power Del., vol. 24, no. 1, pp. 390-399, Jan. 2009.

[44] T. Noda and T. Kikuma, "A robust and efficient iterative scheme for the EMT simulations of nonlinear circuits," IEEE Trans. Power Del., vol. 26, no. 2, pp. 1030-1038, Apr. 2011.

[45] T. Noda, "XTAP," in Numer. Anal. Power Syst. Transients Dyn., A. Ametani, Ed. IET, Jan. 2015, ch. 5, pp. 169-211.

[46] L. O. Chua, C. A. Desoer, and E. S. Kuh, Linear and Nonlinear Circuits. McGraw-Hill, 1987.

[47] M. Günther and U. Feldmann, "CAD-based electric-circuit modeling in industry I. Mathematical structure and index of network equations," Surv. Math. Ind., vol. 8, pp. 97-129, 1999.

[48] K. E. Brenan, S. L. Campbell, and L. R. Petzold, Numerical Solution of Initial-Value Problems in Differential-Algebraic Equations. Siam, 1996, vol. 14.

[49] E. Hairer and G. Wanner, Solving Ordinary Differential Equations II. Springer, 1996.

[50] E. Hairer, S. P. Nørsett, and G. Wanner, Solving Ordinary Differential Equations I. Springer, 1993.

[51] R. Alexander, "Diagonally implicit RungeKutta methods for stiff O.D.E.'s," SIAM J. Numer. Anal., vol. 14, no. 6, pp. 1006-1021, 1977.

[52] D. Estévez Schwarz and C. Tischendorf, "Structural analysis of electric circuits and consequences for MNA," Int. J. Circuit Theory Appl., vol. 28, no. 2, pp. 131-162, Mar. 2000.

[53] A. E. A. Araujo, H. W. Dommel, and J. R. Marti, "Converter simulations with the EMTP: Simultaneous solution and backtracking technique," in Proceedings. Jt. Int. Power Conf. Athens Power Tech, vol. 2. IEEE, 1993, pp. 941-945.

[54] V. Q. Do, D. McCallum, P. Giroux, and B. De Kelper, "A backwardforward interpolation technique for a precise modeling of power electronics in HYPERSIM," in Proc. Int. Conf. Power Syst. Transients, Rio de Janeiro, Brazil, 2001.

[55] K. Strunz, L. Linares, J. R. Marti, O. Huet, and X. Lombard, "Efficient and accurate representation of asynchronous network structure changing phenomena in digital real time simulators," IEEE Trans. Power Syst., vol. 15, no. 2, pp. 586-592, May 2000.

[56] L. R. Linares and J. R. Martí, "A resynchronization algorithm for topological changes in real time fast transients simulation," in Proc. Power Syst. Comput. Conf., Sevilla, Spain, 2002.

[57] K. Strunz, "Flexible numerical integration for efficient representation of switching in real time electromagnetic transients simulation," IEEE Trans. Power Del., vol. 19, no. 3, pp. 1276-1283, Jul. 2004.

[58] J. Tant, W. Leterme, J. Beerten, W. Michiels, and J. Driesen, "Generic circuit partitioning method for efficient simulation of modular multilevel converter topologies," in Proc. 15th IEEE Work. Control Model. Power Electron., Santander, Spain, Jun. 2014, pp. 1-8.

[59] J. Tant and J. Driesen, "Accurate second-order interpolation for power electronic circuit simulation," in Proc. 18th IEEE Work. Control Model. Power Electron., Stanford, CA, Jul. 2017, pp. 1-8.

[60] PLECS User Manual, Plexim GmbH, 2015, version 3.7. [Online]. Available: http://www.plexim.com/plecs 


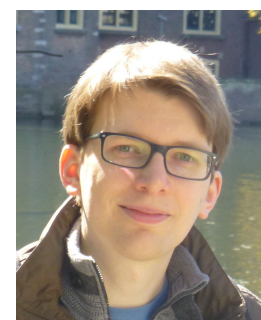

Jeroen Tant (S'08 - M'17) received the M.Sc. degree in Electrical Engineering from the University College Howest, Kortrijk, Belgium, in 2007 and the M.Sc. degree in Mathematical Engineering from KU Leuven, Leuven, Belgium, in 2009. He received the Ph.D. degree in Electrical Engineering, from KU Leuven, in 2017. He held a Ph.D. fellowship from the Research Foundation Flanders (FWO). He is currently working as a postdoctoral researcher at KU Leuven. His research topics include the modeling and simulation of power electronic circuits.

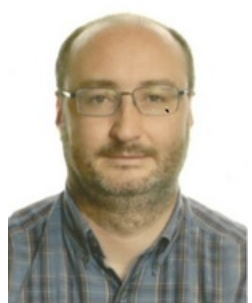

Johan Driesen (S'93 - M'97 - SM'12) received the M.Sc. and Ph.D. degrees in Electrical Engineering from the KU Leuven, Leuven, Belgium, in 1996 and 2000 , respectively. In 2000-2001 he was a visiting researcher in the Imperial College of Science, Technology and Medicine, London, UK. In 2002 he was working at the University of California, Berkeley, USA. Currently he is a full professor at the KU Leuven and teaches power electronics, renewables, drives and electromobility. He conducts research on distributed energy resources, including renewable energy systems, power electronics and its applications. Within EnergyVille, the research collaboration specializing in energy in smart cities and buildings, in cooperation with VITO and Imec, he is involved in the programs on power electronics, distributed energy resources, electric vehicles and storage interfaces. Johan Driesen is also the program director of the international master programs in energy at KU Leuven, and he is the Education Director for the Benelux area within the EIT-KIC InnoEnergy, a pan-European consortium supporting education and innovation in sustainable energy. 